\title{
Clinically significant bleeding in incurable cancer patients: effectiveness of hemostatic radiotherapy
}

\author{
Nikola Cihoric ${ }^{1}$ Susanne Crowe ${ }^{2}$, Steffen Eychmüller ${ }^{3}$, Daniel M Aebersold ${ }^{1}$ and Pirus Ghadjar ${ }^{1 *}$
}

\begin{abstract}
Background: This study was performed to evaluate the outcome after hemostatic radiotherapy (RT) of significant bleeding in incurable cancer patients.

Methods: Patients treated by hemostatic RT between November 2006 and February 2010 were retrospectively analyzed. Bleeding was assessed according to the World Health Organization (WHO) scale (grade $0=$ no bleeding, 1 = petechial bleeding, 2 =clinically significant bleeding, 3 = bleeding requiring transfusion, $4=$ bleeding associated with fatality). The primary endpoint was bleeding at the end of RT. Key secondary endpoints included overall survival (OS) and acute toxicity. The bleeding score before and after RT were compared using the Wilcoxon signed rank test. Time to event endpoints were estimated using the Kaplan Meier method.
\end{abstract}

Results: Overall 62 patients were analyzed including 1 patient whose benign cause of bleeding was pseudomyxoma peritonei. Median age was 66 (range, 37-93) years. Before RT, bleeding was graded as 2 and 3 in 24 (39\%) and 38 (61\%) patients, respectively. A median dose of 20 (range, 5-45) Gy of hemostatic RT was applied to the bleeding site. At the end of RT, there was a statistically significant difference in bleeding $(p<0.001)$; it was graded as $0(n=39), 1(n=12), 2(n=6), 3(n=4)$ and $4(n=1)$. With a median follow-up of 19.3 (range, 0.3-19.3) months, the 6 -month OS rate was 43\%. Forty patients died (65\%); 5 due to bleeding. No grade 3 or above acute toxicity was observed.

Conclusions: Hemostatic RT seems to be a safe and effective treatment for clinically and statistically significantly reducing bleeding in incurable cancer patients.

Keywords: Cancer, Bleeding, Hemostatic, Palliative, Radiotherapy

\section{Background}

Bleeding in cancer patients can occur in a variety of ways, from chronic occult bleeding to clinically significant macroscopic bleeding or profound bleeding from large blood vessels which may cause sudden death. It can be the first symptom of a disease or develop later along with disease progression. It has been estimated that bleeding occurs in approximately $6-10 \%$ of patients with advanced cancer; for at least some of these patients, bleeding will be the direct cause of death [1].

Clinically significant bleeding commonly leads to hospitalization. It is distressing to patients and their families; thus bleeding is likely to impact negatively upon patient's quality of life (QoL). The hemostatic effectiveness

\footnotetext{
* Correspondence: pirus.ghadjar@insel.ch

'Department of Radiation Oncology, Bern University Hospital, and University of Bern, Bern, Switzerland

Full list of author information is available at the end of the article
}

of radiotherapy (RT) is usually visible after only a few fractions of RT and generally explained by increased adhesion of platelets to the vascular endothelium [2]. The long term effect could be explained by causing vessel fibrosis combined with tumor remission [3]. Although RT has been used for decades as a non-invasive treatment for cancer related bleeding, there is little published literature focusing on hemostatic RT. The reduction of hemoptysis achieved by palliative RT in non-small cell lung cancer (NSCLC) has been demonstrated within prospective randomized trials $[4,5]$. Moreover, several relatively small retrospective studies have described the effectiveness of hemostatic RT for vaginal bleeding caused by cervical or endometrial cancer [6,7], bleeding from locally advanced bladder cancer [8], prostate cancer [9], rectal cancer [10] and gastric cancer [11].

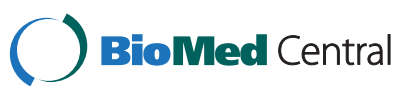

(c) 2012 Cihoric et al.; licensee BioMed Central Ltd. This is an Open Access article distributed under the terms of the Creative Commons Attribution License (http://creativecommons.org/licenses/by/2.0), which permits unrestricted use, distribution, and reproduction in any medium, provided the original work is properly cited. 
This study was performed to evaluate the outcome after hemostatic RT in patients who suffered clinically significant bleeding in different sites, primary tumors or involved metastases.

\section{Methods}

\section{Patient selection}

In this retrospective study all patients who were treated by hemostatic RT, in the Department of Radiation Oncology, Bern University Hospital, Switzerland between November 2006 and February 2010, were selected. All of these patients had advanced incurable cancer; 1 patient's benign cause of bleeding was pseudomyxoma peritonei. Prior surgery, chemotherapy or RT was allowed as well as prior hemostatic measures. Pretreatment investigations included complete medical history, physical examination and radiological or endoscopic examination to confirm or diagnose the bleeding site, if needed. This study was approved by the local ethics committee.

\section{Treatment}

Patients were either treated by external beam RT (EBRT), high-dose rate brachytherapy (HDR-B) monotherapy or a combination of HDR-B and EBRT. EBRT was applied using one of the following methods: a threedimensional conformal RT (3D-CRT), a conventional two-dimensional RT or a volumetric modulated arc technique. For 3D-CRT a dedicated computed tomography $(\mathrm{CT})$ scan was used for treatment planning. All treatment plans were calculated by a dedicated treatment planning system. EBRT was applied with photons from a linear accelerator. RT could also be accompanied by concomitant cisplatin based chemotherapy in certain cases.

Due to the different fractionation schedules used the dose prescriptions were translated into 3-Gy equivalent doses (EQD ${ }_{3}$ ), assuming $\alpha / \beta=10$, according to the linear quadratic model, with no correction for overall treatment time, as a total dose of 30 Gy using daily fractions of $3 \mathrm{~Gy}$ is a widely accepted and commonly used treatment schedule for palliative RT.

\section{Assessment and evaluations}

Patients were initially seen daily by a radiation oncologist during RT, then twice weekly when the bleeding completely stopped. Due to the limited life expectancy of treated patients and their generally poor performance status (PS), patients were commonly followed-up close to their domicile either by their treating medical oncologist or family doctor. Therefore, in most cases, the patients' condition was continuously reported. These reports together with documentation in case of further treatment in Bern University Hospital and additional information provided by the treating medical oncologist or family doctor were used to assess bleeding status at the end of follow-up and overall survival (OS).

The bleeding score was retrospectively assessed by one single observer (NC) using all available clinical information.

Bleeding was assessed before RT, the end of RT and the end of follow-up. It was graded according to the World Health Organization (WHO) scale [12] (grade $0=$ no bleeding, 1 = petechial bleeding, $2=$ clinically significant mild blood loss, $3=$ gross blood loss requiring transfusion, $4=$ debilitating blood loss associated with fatality).

OS was calculated from the last day of RT until death. Patients not experiencing an event were censored at the date of the last follow-up visit.

Toxicities were graded according to the National Cancer Institute Common Terminology Criteria for AEs (CTCAE) version 3.0. Acute toxicity was defined as toxicities occurring during or within three months after completion of RT.

\section{Statistical considerations}

The primary endpoint was bleeding at the end of RT. Secondary endpoints included bleeding at the end of follow-up, OS as well as acute toxicity.

Bleeding before and after RT was compared using the Wilcoxon signed rank test. OS was estimated using the Kaplan Meier (KM) method. The median follow-up time was calculated using the inverse KM method, the respective range is based on patients without an event. Categorical variables were summarized using absolute and relative frequencies; continuous variables by descriptive statistics. To analyse potential associations between bleeding control and clinical variables, the following variables were included: WHO bleeding score at the end of hemostatic $\mathrm{RT}$ and at the end of follow-up (grade $0-1$ vs. grade 2-4), WHO bleeding score before hemostatic RT (grade 2 vs. grade 3 ), age ( $\leq 66$ years vs. $>66$ years), sex, Karnowsky PS (KPS) ( $\geq 50$ vs. $\leq 40$ ), histology (adenocarcinoma vs. others), presence of liver metastasis (no vs. yes), bleeding cause (primary tumor vs. metastasis), use of chemotherapy before hemostatic RT (no vs. yes), other RT prior to hemostatic RT (no vs. yes), time from first diagnosis to hemostatic RT ( $\geq 12$ months vs. $<12$ months), use of any other hemostatic measure (no vs. yes), dose of hemostatic RT ( $<30$ Gy vs. $\geq 30$ Gy), and compared using the Chi-square test. Univariate and multivariate analyses for OS were performed using Cox proportional hazards models and the backward selection method (criterion for removal: $p \geq 0.05$ ). To be assessed in the multivariate analysis, a variable first had to be significant $(\mathrm{p} \leq 0.1)$ in the univariate analysis. The data were analyzed in SPSS (SPSS Inc., Chicago, IL, version 19.0) and SAS (Statistical Analysis Systems Institute Inc, version 9.2). 


\section{Results}

\section{Patient characteristics}

Between November 2006 and February 2010, 62 patients were treated by hemostatic RT. Of these patients, 61 had incurable cancer disease, either due to a locally advanced primary tumor and/or presence of distant metastasis and 1 had pseudomyxoma peritonei. Median age was 66 (range, 37-93) years. Median time from the first diagnosis of the disease until start of hemostatic RT was 11 (range, 0.3-183) months. Before RT, median KPS was 40 (range, 20-80). Initial bleeding was graded as 2 and 3 in $24(39 \%)$ and $38(61 \%)$ patients, respectively. Six patients were lost to follow-up and hence will only be included in the evaluation of the primary endpoint. Further patient characteristics are summarized in Table 1.

\section{Treatment}

Prior to treatment, 8 patients $(13 \%)$ had already undergone RT to the site which was currently bleeding as part of their initial treatment, using a median dose of 61.5 (range, 10-70) Gy. Median time between primary RT and hemostatic RT in these patients was 32 (range, 2$128)$ months. Thirty-three patients $(53 \%)$ received prior chemotherapy; 8 (13\%) of which received chemotherapy within 1 month prior to hemostatic RT. Three patients (5\%) took anticoagulants, of whom 2 continued this medication during and after hemostatic RT. Thirteen patients (21\%) had already undergone the following hemostatic measures prior to hemostatic RT: vaginal tamponade $(n=4)$, cauterization $(n=4)$, tumor resection $(n=2)$, embolization $(n=2)$ and infiltration with adrenaline $(n=1)$. However, these measures were not successful, thus the patients were referred to RT. Hemostatic RT was then given to the bleeding site. Further information on treatment is summarized in Table 2.

Sixty patients (97\%) were treated by EBRT alone using either 3D-CRT $(\mathrm{n}=58)$, conventional two-dimensional RT $(\mathrm{n}=1)$ or a volumetric modulated arc technique $(\mathrm{n}=1)$. The different fractionation schedules used are summarized in Table 3. In 13 patients, the single dose was adapted during RT for a variety of reasons. Median single dose by EBRT for the remaining 47 patients was 3 (range, 2-8) Gy, 1 fraction prescribed per day, 5 days per week. Of the remaining patients, 1 with esophageal cancer was treated by HDR-B monotherapy ( 2 x 5 Gy) and the other with cervical cancer was treated with a combination of HDR-B ( $2 \times 7$ Gy) and 3D-CRT (5 x 4 Gy).

Median total dose to the bleeding site by EBRT (excluding the patient with HDR-B monotherapy) was 20 (range, 5-45) Gy. Median RT duration was 8.5 (range, 1-25) days. RT was accompanied by concomitant cisplatin based chemotherapy in 2 patients (3\%) with NSCLC.

\section{Bleeding control at the end of RT}

At the end of RT, the following bleeding grades were observed in patients: $0(n=39), 1 \quad(n=12), 2 \quad(n=6), 3$ $(n=4)$ and $4(n=1)$. It should be noted that 8 patients with either grade 2 or 3 at the end of RT were treated with less than $30 \mathrm{~Gy}$. The incidence of bleeding was statistically significantly different from the bleeding status prior to hemostatic RT $(p<0.001)$ (Figure 1).

Bleeding was improved in 54 patients (87\%); 39 (63\%) of whom had a complete response (CR) to bleeding. Of the remaining patients, bleeding was unchanged in 7 patients $(11 \%)$ whilst 1 patient showed a progression in bleeding (Figure 2). This elderly gastric cancer patient died as a result of bleeding, after completion of 5 fractions reaching a total dose of 9.4 Gy (first fraction 1.4 Gy due to technical problems, followed by $4 \times 2$ Gy). The patient was originally referred to RT after repeated non-successful cauterizations with grade 3 bleeding.

Using the Chi-square test, sex was found to be associated with worse bleeding control at the end of hemostatic RT $(p=0.0253)$. However, age, KPS, histology, presence of liver metastasis, use of chemotherapy or other RT prior to hemostatic RT, time from first diagnosis to hemostatic RT, bleeding cause (primary tumor vs. metastasis), WHO bleeding score before hemostatic RT, use of any other hemostatic measure and dose of hemostatic RT (<30 Gy vs. $\geq 30$ Gy) were not significantly associated with bleeding control at the end of RT. When the response of bleeding at the end of RT was analyzed according to the respective bleeding site, it indicated that lesions in the lung (100\% improved), uterovaginal lesions (95\% improved) and upper gastrointestinal (GI) lesions (90\% improved) showed a better response than lesions involving the bladder $(65 \%$ improved) (Table 4).

\section{Bleeding control at end of follow-up}

At the end of follow-up, the incidence of bleeding was as follows: grade $0(n=40)$, grade $1(n=5)$, grade 2 $(n=1)$, grade $3(n=5)$ and grade $4(n=5)$. This was statistically significantly different from the bleeding status prior to hemostatic RT $(p<0.001$, worse $=6$, no change $=$ 5 , improved $=45)($ Figure 1).

The following grades were observed at the end of follow-up for the 39 patients with no bleeding (grade 0 ) at the end of RT: $0(n=26), 1(n=2), 2(n=1), 3(n=1)$ and $4(n=2)$. At the end of follow-up, the 12 patients with grade 1 bleeding at the end of RT either had grade $0(n=11)$ or $1(n=1)$ bleeding.

From end of RT to end of follow-up, for those who could be assessed, the grade worsened in 9 patients, remained unchanged in 33 and improved in 14 (Figure 2). The change in grade in bleeding scores at end of follow-up from the end of RT were analyzed 


\section{Table 1 Patient Characteristics}

Characteristics ( $\mathrm{N}=62$ )

Age (years)

Median (range) 66 (37-93)
Sex
Female
Male

Karnowsky PS

Median (range)

$\leq 40$

$\geq 50$

Site*

Bladder

Lung (NSCLC)

Endometrial

Prostate

Cervical

Gastric

Ovarial

Colorectal

Others $^{\#}$

Histology ${ }^{+}$

Adenocarcinoma

Squamous cell carcinoma

Transitional cell carcinoma

Others

Tumor-classification $^{+}$

$\begin{array}{ll}\text { T0 } & 2(3) \\ \text { T3 } & 2(3) \\ \text { T4 } & 55(94)\end{array}$

Metastasis ${ }^{+}$

No

Yes

Unknown

Liver metastasis ${ }^{+}$

No

Yes

Bleeding Site ${ }^{+}$

Primary tumor

Metastasis

Symptom

Vaginal bleeding

Hematuria

Hemoptysis

Others n (\%)

$40(20-80)$

32 (52)

30 (48)

10 (16)

9 (15)

8 (13)

6 (10)

$6(10)$

6 (10)

$6(10)$

3 (5)

8 (13)

$38(65)$

6 (10)

9 (15)

6 (10)

2 (3)

2 (3)

55 (94)

1 (2)

52 (88)

6 (10)

43 (73)

16 (27)

48 (81)

11 (19)

19 (31)

17 (27)

10 (16)

16 (26)
Table 1 Patient Characteristics (Continued)

First diagnosis to hRT (months)

$$
\text { Median (range) } 11 \text { (0.3-183) }
$$

Abbreviations: $P S=$ performance status; $N S C L C=$ non-small cell lung cancer; $h R T=$ hemostatic radiotherapy; ${ }^{\text {SSite }}$ of the primary tumor; ${ }^{*}$ including esophageal $(n=2)$, sarcoma $(n=1)$, multiple myeloma $(n=1)$, kidney cancer $(n=1)$, non-hodgkin lymphoma $(n=1)$, breast cancer $(n=1)$ and pseudomyxoma peritonei $(n=1) ;{ }^{+}$Three patients were not applicable having stage IV non-hodgkin lymphoma according to Ann Arbor $(n=1)$ or stage III multiple myeloma according to Durie and Salmon $(n=1)$ or benign pseudomyxoma peritonei $(n=59)$.

according to the respective bleeding site. Lesions involving the bladder ( $29 \%$ worsened) and upper GI ( $20 \%$ worsened) appeared to have worse scores than lung $(0 \%$ worsened) or uterovaginal lesions $(10.5 \%$ worsened $)$ (Table 4).

\section{Table 2 Treatment characteristics}

\begin{tabular}{ll}
\hline Characteristics $(\mathbf{N}=\mathbf{6 2})$ & $\mathbf{n}(\mathbf{\%})$ \\
\hline Chemotherapy before hRT & \\
No & $29(47)$ \\
Yes & $33(53)$
\end{tabular}

Time last ChT to hRT (months)

$$
\text { Median (range) } 3(0.2-43)
$$

Chemotherapy during hRT

No

Yes

Other RT prior hRT*

$$
\text { Yes }
$$

Time last RT to hRT (months)

$$
\text { Median (range) } \quad 32(2-128)
$$

Other hemostatic measure

$$
\begin{array}{ll}
\text { No } & 49(79) \\
\text { Yes } & 13(21)
\end{array}
$$

Total hRT dose (Gy)

$$
\text { Median (range) }
$$

$20(5-45)$

Region treated by hRT

$$
\begin{array}{lc}
\text { Uterovaginal } & 19(31) \\
\text { Bladder } & 17(27) \\
\text { Lung } & 10(16) \\
\text { Upper Gl } & 10(16) \\
\text { Other }^{\#} & 6(10)
\end{array}
$$

Treatment time hRT (days)

Median (range)

$8(1-25)$

Abbreviations: $R T=$ radiotherapy; $h R T=$ hemostatic radiotherapy; $C h T=$ chemotherapy; $G l=$ gastrointestinal; ${ }^{*}$ in the same region as hemostatic $R T$; "including lower gastrointestinal $(n=4)$, skin $(n=1)$ and abdominal wall $(n=1)$. 
Table 3 External beam radiotherapy fractionation schedules and the respective 3-Gy equivalent doses

\begin{tabular}{|c|c|c|c|}
\hline $\begin{array}{l}\text { Fractionation } \\
\text { schedule }\end{array}$ & $\begin{array}{l}\text { Number of patients } \\
\text { in each schedule } \\
(\mathrm{N}=47)^{*} \mathrm{n}(\%)\end{array}$ & $\begin{array}{l}\text { Total dose } \\
\text { (Gy) }\end{array}$ & $\begin{array}{c}\text { 3-Gy ED (Gy) } \\
(\alpha / \beta=10)\end{array}$ \\
\hline 10 x 2 Gy & $5(11 \%)$ & 20 & 18.5 \\
\hline 15 x 2 Gy & $2(4 \%)$ & 30 & 27.7 \\
\hline 12 x 2 Gy & $1(2 \%)$ & 24 & 22.1 \\
\hline $18 \times 2.5 \mathrm{~Gy}$ & $2(4 \%)$ & 45 & 43.3 \\
\hline 16 × $2.5 \mathrm{~Gy}$ & $1(2 \%)$ & 40 & 38.5 \\
\hline 10 x 3 Gy & 10 (21\%) & 30 & 30.0 \\
\hline 13 x 3 Gy & $2(4 \%)$ & 39 & 39.0 \\
\hline 5 x 3 Gy & $2(4 \%)$ & 15 & 15.0 \\
\hline 9 x 3 Gy & $1(2 \%)$ & 27 & 27.0 \\
\hline 4 x 3 Gy & $1(2 \%)$ & 12 & 12.0 \\
\hline 5 x 4 Gy & $12(26 \%)$ & 20 & 21.5 \\
\hline 4 x 4 Gy & $2(4 \%)$ & 16 & 17.2 \\
\hline 6 x $4 \mathrm{~Gy}$ & $1(2 \%)$ & 24 & 25.8 \\
\hline 3 x 4 Gy & $1(2 \%)$ & 12 & 12.9 \\
\hline 5 x 5 Gy & $1(2 \%)$ & 25 & 28.8 \\
\hline 4 x 5 Gy & $1(2 \%)$ & 20 & 23.1 \\
\hline 1 x 5 Gy & $1(2 \%)$ & 5 & 5.8 \\
\hline $1 \times 8 \mathrm{~Gy}$ & $1(2 \%)$ & 8 & 11.1 \\
\hline
\end{tabular}

Abbreviations: $E D=$ equivalent dose; ${ }^{*}$ in 13 patients the single dose was adapted during RT and 2 patients underwent high-dose rate brachytherapy.

\section{Overall survival}

Four patients (6\%) died during RT when the prescribed dose was not completely delivered for the following

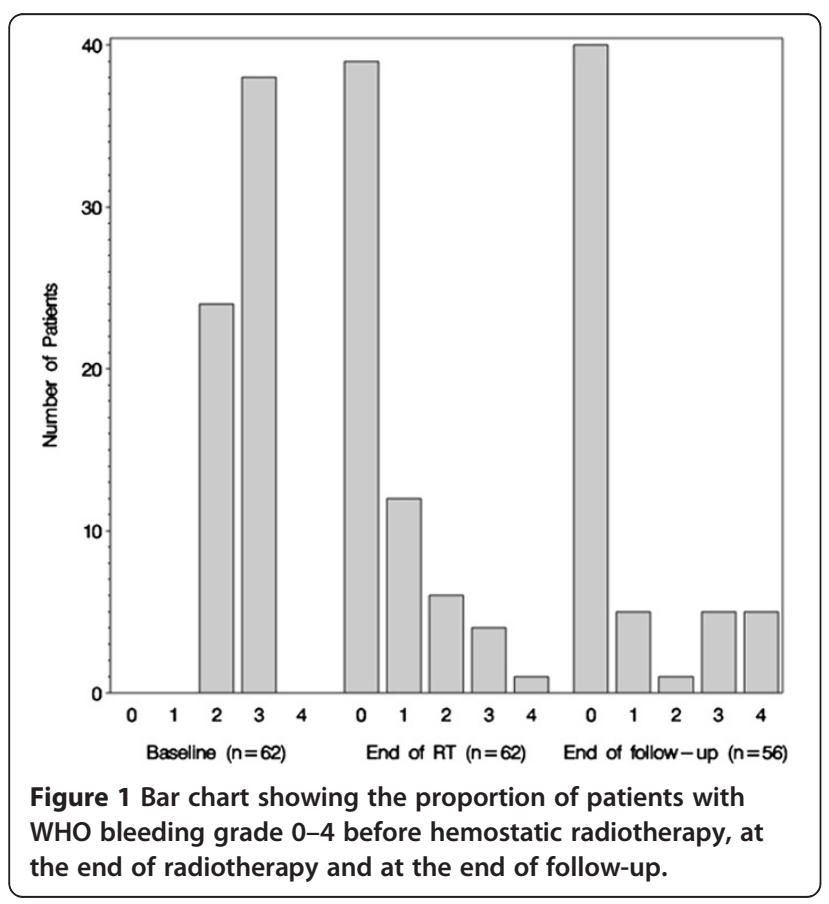

reasons: bleeding (grade 4) from the irradiated volume $(\mathrm{n}=1)$, kidney failure $(\mathrm{n}=2)$ and septicemia $(\mathrm{n}=1)$. A further 36 patients (58\%) died during follow-up; 4 (6\%) of which were attributed to bleeding from the treated site. Median time to death based on data from all patients was 2.4 (range, 0.03-30.9) months.

With a median follow-up of 19.3 (range, 0.3-19.3) months, the 6-month, 1-year and 2-year OS rates were $43 \%, 24 \%$ and $7 \%$, respectively (Figure 3 ).

In the univariate Cox proportional hazards model, sex was associated with OS (hazard ratio (HR): 1.967; 95\% Confidence interval (CI): 1.033-3.748; $p=0.0396)$; male patients have a greater risk of dying. RT dose was also associated with OS (HR: 2.669; 95\% CI: 1.2865.540; $p=0.0084)$; patients who receive a low RT dose $(<30$ Gy) are more likely to die. This was also the case when the same dose cut-off was used based on the $\mathrm{EQD}_{3}$ (HR: 2.621; 95\% CI: 1.228-5.592; $p=0.0127$ ). Furthermore, bleeding grade at the end of RT was associated with OS (HR: 5.882; 95\% CI: 2.445-13.865; $p<0.0001$ ), patients with bleeding grade $\geq 2$ are shown to have a greater risk of dying. In the multivariate analysis higher bleeding grade at the end of RT and lower RT dose remained statistically significantly associated with decreased OS (Table 5).

\section{Acute toxicity}

Six patients (10\%) experienced grade 1 acute toxicity; observed symptoms included diarrhea, esophagitis, abdominal pain and vaginitis. Three patients (5\%) had grade 2 acute toxicity with symptoms such as esophagitis, nausea and erythema. No grade 3 or higher acute toxicities were observed.

\section{Discussion}

We are not currently aware of any established standard therapeutic approach for the treatment of advanced cancer patients with clinically significant bleeding as a result of their cancer. At the moment, treatment decisions are primarily based on the underlying causes and overall patient PS. The therapeutic approach varies from comfort measurement to invasive surgical procedures.

Hemostatic RT is generally believed to be an effective treatment for patients with bleeding due to cancer. Although bleeding occurs in $6-10 \%$ of patients with advanced cancer [1], relatively little published literature is available which focuses on bleeding control of hemostatic RT; even less is known about the optimal RT total dose and fractionation.

In our retrospective study containing 62 patients with different primary tumors with significant bleeding (including 1 patient whose benign cause of bleeding was pseudomyxoma pertinonei), it was demonstrated that hemostatic RT reduced the incidence and grading of 

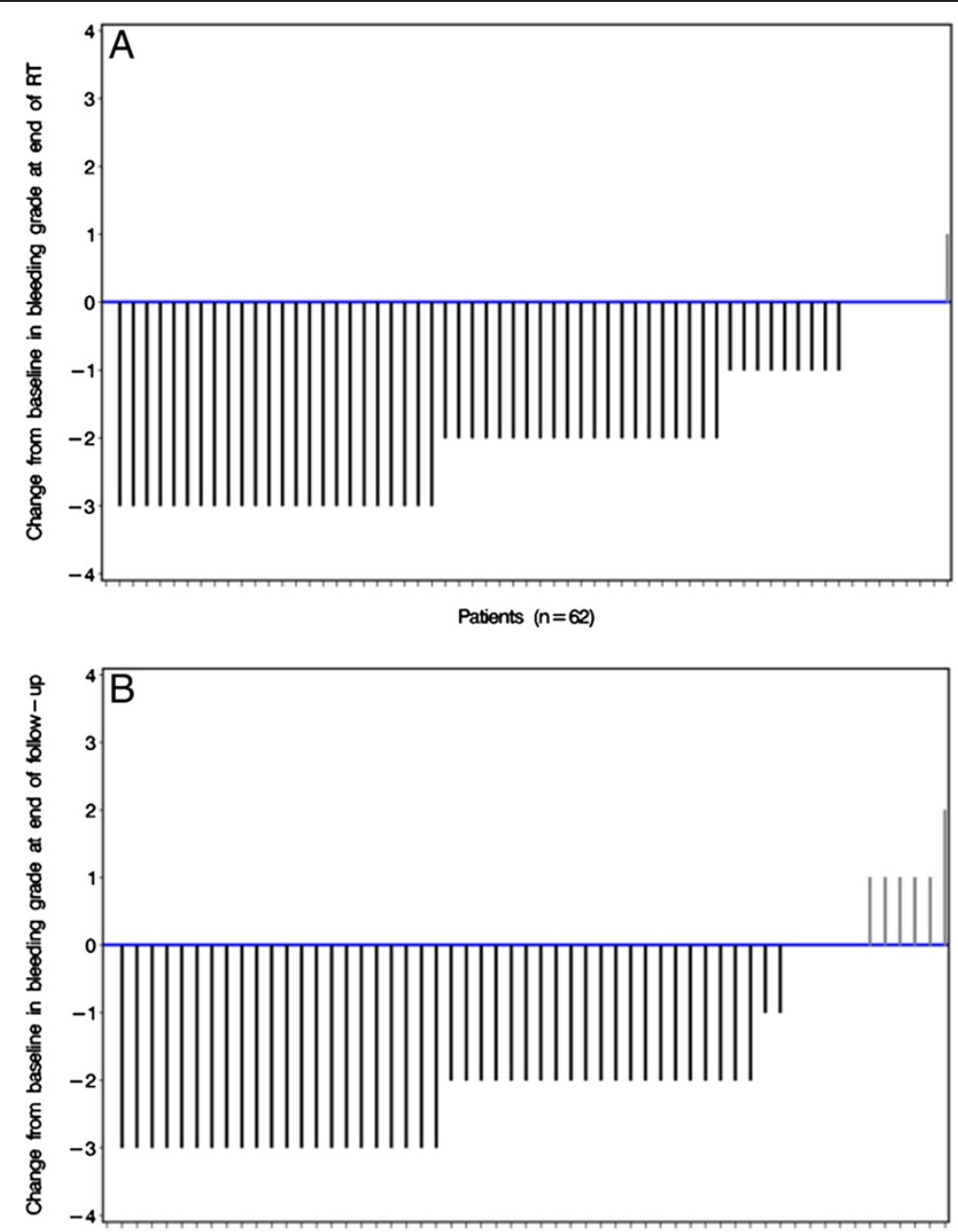

Patients $(n=56)$

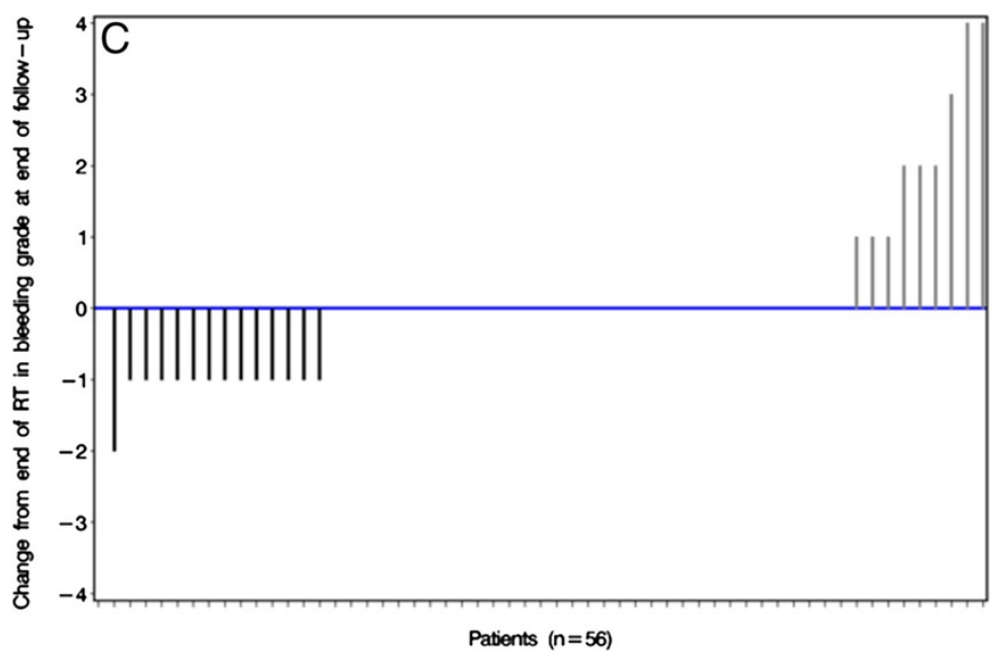

Figure 2 Waterfall plot showing change in bleeding grade at end of RT as compared to baseline (A), change in bleeding grade at end of follow-up as compared to baseline (B) and change in bleeding grade at end of follow-up as compared to at end of RT (C), $<0=$ Improvement; $0=$ Stable (no change); $>0=$ Worsened. 
Table 4 Bleeding control by treated site

\begin{tabular}{|c|c|c|c|c|}
\hline Treated Site (N) & $\begin{array}{l}\text { Change in } \\
\text { grade }\end{array}$ & $\begin{array}{l}\text { Baseline to End } \\
\text { of RT } n(\%)\end{array}$ & $\begin{array}{l}\text { Baseline to End of } \\
\text { follow-up n (\%) }\end{array}$ & $\begin{array}{l}\text { End of RT to End of } \\
\text { follow-up } n(\%)\end{array}$ \\
\hline \multirow[t]{4}{*}{ Uterovaginal $(\mathrm{N}=19)$} & Worsened & - & $1(5.3 \%)$ & $2(10.5 \%)$ \\
\hline & No change & $1(5.3 \%)$ & $1(5.3 \%)$ & $10(52.6 \%)$ \\
\hline & Improved & $18(94.7 \%)$ & 15 (78.9\%) & $5(26.3 \%)$ \\
\hline & Missing & - & $2(10.5 \%)$ & $2(10.5 \%)$ \\
\hline \multirow[t]{4}{*}{ Bladder $(\mathrm{N}=17)$} & Worsened & - & $2(11.8 \%)$ & $5(29.4 \%)$ \\
\hline & No change & $6(35.3 \%)$ & $4(23.5 \%)$ & $8(47.1 \%)$ \\
\hline & Improved & $11(64.7 \%)$ & $10(58.8 \%)$ & $3(17.6 \%)$ \\
\hline & Missing & - & $1(5.9 \%)$ & $1(5.9 \%)$ \\
\hline \multirow[t]{4}{*}{ Lung $(\mathrm{N}=10)$} & Worsened & - & - & - \\
\hline & No change & - & - & $6(60.0 \%)$ \\
\hline & Improved & $10(100.0 \%)$ & $8(80.0 \%)$ & $2(20.0 \%)$ \\
\hline & Missing & - & $2(20.0 \%)$ & $2(20.0 \%)$ \\
\hline \multirow[t]{4}{*}{ Upper Gl (N=10) } & Worsened & $1(10.0 \%)$ & $3(30.0 \%)$ & $2(20.0 \%)$ \\
\hline & No change & - & - & $4(40.0 \%)$ \\
\hline & Improved & $9(90.0 \%)$ & $6(60.0 \%)$ & $3(30.0 \%)$ \\
\hline & Missing & - & $1(10.0 \%)$ & $1(10.0 \%)$ \\
\hline \multirow[t]{3}{*}{ Other $(\mathrm{N}=6)^{*}$} & Worsened & - & - & - \\
\hline & No change & - & - & $5(83.3 \%)$ \\
\hline & Improved & $6(100.0 \%)$ & $6(100.0 \%)$ & 1 (16.7\%) \\
\hline
\end{tabular}

Abbreviations: ${ }^{*}$ including lower gastrointestinal $(n=4)$, skin $(n=1)$ and abdominal wall $(n=1)$.

bleeding significantly. This effect seemed to be durable in the majority of patients. Furthermore, our data suggests an association between a total RT dose $>30$ Gy and increased OS. Male patients and those with a bleeding grade $\geq 2$ at the end of RT were shown to have a greater risk of dying. Although RT dose remained significantly associated with OS in the multivariate analysis, we are aware that this association may be biased due to possible confounding factors, such as increased dose given to patients with a higher PS and/or better prognosis.

Bleehen et al. reported almost 20 years ago results from a phase III trial which compared 2 different fractionation schedules $(1 \times 10$ Gy vs. 2 × 8.5 Gy) as palliative treatment in 235 patients with incurable locally advanced NSCLC. One of the symptoms of interest was hemoptysis which was present in some form in $47 \%$ of

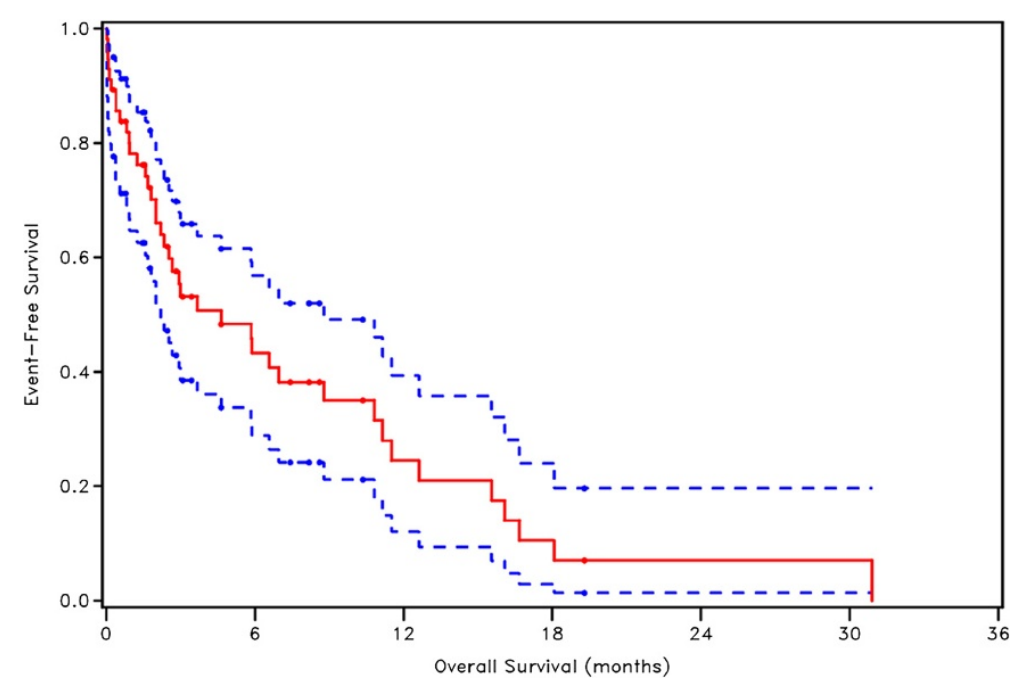

Figure 3 Kaplan Meier plot of overall survival with 95\% confidence interval. 
Table 5 Overall survival analysis

\begin{tabular}{|c|c|c|c|}
\hline \multirow[t]{2}{*}{ Dichotomized variables } & \multirow[t]{2}{*}{ Associated level } & \multicolumn{2}{|l|}{ os } \\
\hline & & Hazard ratio $(95 \% \mathrm{Cl})$ & p-value \\
\hline \multicolumn{4}{|l|}{ Univariate analysis } \\
\hline Age & $>66$ years & $0.971(0.514-1.834)$ & 0.9288 \\
\hline Sex & Male & $1.967(1.033-3.748)$ & 0.0396 \\
\hline KPS & $>50$ & $0.539(0.283-1.029)$ & 0.0611 \\
\hline Histology & Adenocarcinoma & $0.965(0.510-1.825)$ & 0.9118 \\
\hline Presence of liver metastasis & Yes & $0.982(0.463-2.082)$ & 0.9616 \\
\hline Use of chemotherapy before hRT & Yes & $1.085(0.572-2.057)$ & 0.8034 \\
\hline Time from first diagnosis to hRT & $\geq 12$ months & $0.804(0.424-1.524)$ & 0.5037 \\
\hline Other RT prior to hRT & Yes & $1.411(0.656-3.034)$ & 0.3785 \\
\hline Use of any other hemostatic measure & Yes & $1.056(0.409-2.724)$ & 0.9105 \\
\hline Bleeding cause & Primary tumor & $1.420(0.623-3.236)$ & 0.4036 \\
\hline WHO bleeding score before hRT & Grade 3 & $1.380(0.728-2.617)$ & 0.3239 \\
\hline Dose of hRT & $<30$ Gy & $2.669(1.286-5.540)$ & 0.0084 \\
\hline WHO bleeding score at end of hRT & Grade 2-4 & $5.882(2.445-13.865)$ & $<0.0001$ \\
\hline WHO bleeding score at end of follow-up & Grade 2-4 & $2.006(0.969-4.154)$ & 0.0609 \\
\hline \multicolumn{4}{|l|}{ Multivariate analysis } \\
\hline Sex & Male & $1.007(0.487-2.081)$ & 0.9859 \\
\hline Dose of hRT & $<30$ Gy & $2.853(1.360-5.987)$ & 0.0056 \\
\hline WHO bleeding score at end of hRT & Grade 2-4 & $6.456(2.645-16.202)$ & $<0.0001$ \\
\hline
\end{tabular}

Abbreviations: $R T=$ radiotherapy; $h R T=$ hemostatic radiotherapy; $K P S=$ Karnowsky performance score; OS=overall survival.

all patients. After thoracic RT, hemoptysis improved in $72-75 \%$ of patients and completely disappeared in 54$64 \%$ of patients. Median duration of this palliative effect was 64-73 days; no significant difference was observed between the 2 arms [4]. Similarly, Langendijk et al. prospectively assessed the influence of palliative thoracic RT using $10 \times 3$ Gy in 65 patients with incurable NSCLC on QoL. There was a 79\% response rate of hemoptysis after RT [5]. Comparably, the symptoms of all 10 patients where lung was the treated site in our retrospective study improved during RT. There was a $80 \%$ CR rate for bleeding. Furthermore, bleeding remained improved in $80 \%$ of patients until the end of follow-up.

Biswal et al. reported a bleeding control of 100\% 1248 hours after EBRT with 5-20 Gy with/or low-dose rate brachytherapy with 30 Gy for severe refractory bleeding caused by cervical cancer. However, in $85 \%$ bleeding was observed again within the following two years. GI toxicities $\geq$ grade 2 were observed in 3 patients [7]. Of the 19 patients who received RT to the uterovaginal region in our study, improvement of bleeding during RT occurred in 95\%, 68\% had a CR and bleeding remained improved until the end of follow-up for $79 \%$. Srinivasan et al. treated in total 41 cT3-4 patients, with bladder cancer who had hematuria, with $2 \mathrm{x}$ 8.5 Gy $=17$ Gy $\left(\mathrm{EQD}_{3}=24 \mathrm{~Gy}\right)$ as compared to $12 \mathrm{x}$ $3.75 \mathrm{~Gy}=45 \mathrm{~Gy}$. Interestingly, the effect on hematuria was larger in the 17 Gy hypofractionated patients with a $59 \%$ clearing probability as compared to $16 \%$ in the other group, while toxicity did not differ between the groups [8]. Others have reported that 6 weeks after palliative RT using 20 Gy in 5 fractions, the bleeding response rate was $81 \%$ in 31 patients with castrant resistant prostate cancer, however the response rate appeared to drop to $42 \%$ and $29 \%$ after 4 months and 7 months, respectively [9]. Seventeen patients in our study received $\mathrm{RT}$ to the bladder region (for different primary tumors and histologies, respectively); 65\% showed an improvement in their bleeding during RT, 47\% had a CR and 59\% remained with improved symptoms until the end of follow-up.

Hoskin et al. described the outcome after intraluminal HDR-B for 50 patients with inoperable cancer of the rectum or anal canal. Treatment was either performed in curative intent with 6 × 6 Gy = 36 Gy HDR-B monotherapy, 12 Gy HDR-B boost after 45 Gy EBRT $(n=22)$, or 1 x 10 Gy HDR-B as palliative treatment $(n=28)$. Twenty-eight patients had initial bleeding; a 57\% CR rate was obtained in these patients, with a 10-month median response duration [10]. In our study only 4 patients with tumors of the lower GI tract were available, all of whom experienced CR for bleeding which remained until the end of follow-up.

Hemostatic RT was also used to treat locally advanced or recurrent gastric cancer. Tey et al.'s study contained 
33 patients; 24 of whom had initial bleeding. After RT where $10 \times 3$ Gy was applied in the majority of cases, $54 \%$ showed a reduction in bleeding with median response duration of 140 days. No dose-response relationship was observed. One patient experienced grade 3 toxicity [11]. In our study, 10 patients received RT to the upper GI tract; 5 of whom was to the stomach. A reduction in bleeding was observed in $90 \%, \mathrm{CR}$ in $50 \%$ and durable response in $60 \%$. Notably in $30 \%$ of these patients bleeding worsened during the end of follow-up.

We are aware of the limitations within our retrospective non-randomized study; no firm conclusions can be made in regard to the optimal radiation dose. The possible advantages of using a higher RT dose should be balanced against potentially higher toxicity in addition to patient discomfort due to the prolonged treatment time. Hypofractionation with large single doses may be useful in case of life-threatening bleeding to induce rapid hemostypsis, but the risks of severe toxicities (Grade 3-5) have to be taken into account even in this palliative end-of-life setting [13].

We also acknowledge the heterogeneity of our patient population; several different primary tumors were involved and ultimately different treatment sites were targeted by hemostatic RT. While, the total response rate compares well with the current literature for different individual disease types, the bleeding control seemed to be lower in lesions involving the bladder as compared to the other treated sites. This finding, however, is again subject to several potential confounding factors such as use of different total doses and therefore needs further confirmation by other studies.

\section{Conclusions}

Our data focusing on bleeding control in advanced cancer patients strongly suggests that hemostatic RT is a very effective treatment for significantly reducing bleeding of various primary tumors and treatment sites without major toxicity.

\footnotetext{
Abbreviations

$\mathrm{Cl}$ : Convidence interval; CR: Complete response; CT: Computed tomography; CTCAE: Common Terminology Criteria for AE; EBRT: External beam radiotherapy; $\mathrm{EQD}_{3}$ : 3-Gy equivalent doses; Gl: Gastrointestinal; HDR-B: Highdose rate brachytherapy; HR: Hazard ratio; KM: Kaplan Meier; KPS: Karnowsky performance status; NSCLC: Non-small cell lung cancer; OS: Overall survival; QoL: Quality of life; RT: Radiotherapy; PS: Performance status; HRT: Hemostatic radiotherapy; WHO: World Health Organization; 3DCRT: Three-dimensional conformal radiotherapy.
}

\section{Competing interests}

The authors declare that they have no competing interests.

\section{Authors' contributions}

Each author had participated sufficiently in the work to take public responsibility for appropriate portions of the content. NC, PG and DMA designed the study. SC and PG performed the statistical analysis. NC and PG collected the data and together with SE and DMA interpreted the data. The manuscript was written by NC and PG; all other authors helped. All authors have read and approved this manuscript.

\section{Author details}

${ }^{1}$ Department of Radiation Oncology, Bern University Hospital, and University of Bern, Bern, Switzerland. ${ }^{2}$ SAKK Coordinating Center, Bern, Switzerland. ${ }^{3}$ Center of Palliative Care, Bern University Hospital, and University of Bern, Bern, Switzerland.

\section{Received: 3 May 2012 Accepted: 27 July 2012}

Published: 3 August 2012

\section{References}

1. Pereira J, Phan T: Management of bleeding in patients with advanced cancer. Oncologist 2004, 9:561-570.

2. Verheij M, Dewit LG, Boomgaard MN, Brinkman HJ, van Mourik JA: lonizing radiation enhances platelet adhesion to the extracellular matrix of human endothelial cells by an increase in the release of von Willebrand factor. Radiat Res 1994, 137:202-207.

3. Yarnold J, Brotons MC: Pathogenetic mechanisms in radiation fibrosis. Radiother Oncol 2010, 97:149-161.

4. Bleehen NM, Girling DJ, Machin D, Stephens RJ: A Medical Research Council (MRC) randomised trial of palliative radiotherapy with two fractions or a single fraction in patients with inoperable non-small-cell lung cancer (NSCLC) and poor performance status. Br J Cancer 1992, 65:934-941.

5. Langendijk JA, Ten Velde GPM, Aaronson NK, De Jong JMA, Muller MJ, Wouters EFM: Quality of life after palliative radiotherapy in non-small cell lung cancer: A prospective study. Int J Radiat Oncol Biol Phys 2000, 47:149-155.

6. Halle JS, Rosenman JG, Varia MA, Fowler WC, Walton LA, Currie JL: 1000 cGy single dose palliation for advanced carcinoma of the cervix or endometrium. Int J Radiat Oncol Biol Phys 1986, 12:1947-1950.

7. Biswal BM, Lal P, Rath GK, Mohanti BK: Hemostatic radiotherapy in carcinoma of the uterine cervix. Int J Gynaecol Obstet 1995, 50:281-285.

8. Srinivasan V, Brown CH, Turner AG: A comparison of two radiotherapy regimens for the treatment of symptoms from advanced bladder cancer. Clin Oncol (R Coll Radiol) 1994, 6:11-13.

9. Din OS, Thanvi N, Ferguson CJ, Kirkbridge P: Palliative prostate radiotherapy for symptomatic advanced prostate cancer. Radiother Oncol 2009, 93:192-196.

10. Hoskin PJ, de Canha SM, Bownes P, Bryant L, Glynne Jones R: High dose rate afterloading intraluminal brachytherapy for advanced inoperable rectal carcinoma. Radiother Oncol 2004, 73:195-198.

11. Tey J, Back MF, Shakespeare TP, Mukherjee RK, Lu JJ, Lee KM, Wong LC, Leong CN, Zhu M: The role of palliative radiation therapy in symptomatic locally advanced gastric cancer. Int I Radiat Oncol Biol Phys 2007, 67:385-388.

12. Miller $A B$, Hoogstraten $B$, Staquet $M$, Winkler $A$ : Reporting results of cancer treatment. Cancer 1981, 47:207-214.

13. Yan J, Milosevic M, Fyles A, Manchul L, Kelly V, Levin W: A hypofractionated radiotherapy regimen (0-7-21) for advanced gynaecological cancer patients. Clin Oncol 2011, 23:476-481.

doi:10.1186/1748-717X-7-132

Cite this article as: Cihoric et al: Clinically significant bleeding in incurable cancer patients: effectiveness of hemostatic radiotherapy. Radiation Oncology 2012 7:132. 